\title{
Analysis of the Effect of Magnetic Resonance Diffusion Tensor Imaging in Patients with Stuttering
}

\author{
Wenjing Ge \\ Qingdao Municipal Hospital, Qingdao 266071, Shandong, China \\ Email: smartleng@163.com
}

\begin{abstract}
Purpose - To discuss the applying effectiveness analysis of magnetic resonance diffusion tensor imaging among stuttering patients. Method — Choose 81 suspected stuttering patients between May, 2018 to May, 2021 as researching objects. All of the patients did the test of magnetic resonance diffusion tensor imaging. And then the testing results was compared with clinical examination results (Gold standard), ROC curve was drawn to analyze the applying effectiveness analysis of magnetic resonance diffusion tensor imaging among stuttering patients. Results - 70 patients were diagnosed as stuttering patients from clinical examination among the 81 suspected stuttering patients. The diagnosis rate is $86.42 \%$. After all of the patients did the test of magnetic resonance diffusion tensor imaging, 68 patients were diagnosed as stuttering patients finally. Diagnosis coincidence rate is $97.14 \%(\mathrm{P}>0.05)$. The rFA levels of the insula, corpus callosum, and putamen on the side of stuttering lesion are lower than those on the healthy side $(\mathrm{P}<0.05)$. The rFA of the cerebrum, internal capsule, and cerebellum on the lesion side and the contralateral side has no statistical significance $(\mathrm{P}>0.05)$. The ROC curve shows that the AUC data of magnetic resonance diffusion tensor imaging in stuttering patients is 0.834 , the diagnostic sensitivity is $84.31 \%$, and the specificity is $71.48 \%$. Conclusion - Magnetic resonance diffusion tensor imaging applying in stuttering patients can gain higher detection rate, which can provide imaging evidence for clinical diagnosis and treatment for clinical and is worthy to promoting and applying.
\end{abstract}

Keywords: magnetic resonance diffusion tensor imaging, stuttering symptom, application effect, ROC curve, application efficiency

\section{Introduction}

Stuttering belongs to speech disorder. The stuttering patients behave as the speech of frequency and intensity of the patients is different from that of normal people, and their speech is repeated, paused or prolonged and interrupted unwillingly ${ }^{[1]}$. Previous studies have shown that many manifestations of stuttering are difficult to be observed by other people, such as fear of specific phonemes, characters and words, fear and anxiety of specific situations and so on ${ }^{[2]}$. At present, the pathogenesis of stuttering has not been clarified clinically, and it is generally thought to be related to genetics, neurophysiological development, family, society and the other factors. Therefore, strengthening the diagnosis and treatment of stuttering patients is of great significance for improving the prognosis of patients ${ }^{[3]}$. Magnetic resonance diffusion tensor imaging is a new method to describe the structure of the brain. It can quantitatively evaluate the anisotropy of the white matter of the brain. It analyzes the diffusion of water molecules in a three-dimensional space and requires to impose a sensitive gradient magnetic field in multiple directions on the spatial coordinate axis. Its imaging principle is the same as that of diffusion-weighted imaging in different directions ${ }^{[4]}$. However, there are few researches on the application of Magnetic resonance diffusion tensor imaging in patients with stuttering. Therefore, this study chose the stuttering patients as studying object, and explored the application effect of Magnetic resonance diffusion tensor imaging in patients with stuttering. The report is as follows:

\section{Materials and methods}

\subsection{Clinical materials}

Choose 81 suspected stuttering patients between May, 2018 to May, 2021 as researching object: 45 men, 36 women, 21-56 years old, $41.59 \pm 4.62$ years old on average; sick period (1-8) years, $4.34 \pm 0.51$ years on average; body mass index (BMI) $(18-29) \mathrm{kg} / \mathrm{m}^{2}, 23.51 \pm 3.49 \mathrm{~kg} / \mathrm{m}^{2}$ on average. Inclusion criteria: (1) meeting the diagnostic criteria for stuttering, all diagnosed by clinical examination ${ }^{[5]}$; (2) both can complete the magnetic resonance diffusion tensor imaging examination, and the patient can tolerate it; (3) having complete baseline and follow-up data. exclusion criteria: (1) patients with mental 
disorders, organic diseases or abnormal blood coagulation function; (2) patients with blood system diseases or serious liver and kidney dysfunction.

\subsection{Methods}

\subsubsection{Testing methods}

All patients were diagnosed as stuttering by clinical examination. Before the diagnosis, the patients got magnetic resonance diffusion tensor imaging test. The specific methods are as follows.

The patient was examined with a $1.5 \mathrm{~T}$ superconducting magnetic resonance scanner produced by GE in the United States. Magnetic resonance diffusion tensor imaging adopts single-shot planar echo technology, and the relevant parameters are set according to the examination site. The repetition time (TR) is $10000 \mathrm{~ms}$, the echo time (TE) is $115 \mathrm{~ms}$, the number of excitations is 2, the setting matrix is $256 \times 256$, the field of view is $256 \mathrm{~mm}$, the spatial resolution is $1.0 \mathrm{~mm} \times 1.0 \mathrm{~mm} \times 3.0 \mathrm{~mm}$, and 40 standard axes images are completed. It spreads diffusion sensitive gradients from 15 directions respectively, sets value $=1000 \mathrm{~s} / \mathrm{mm}^{2}$. It has 8 -channel head coil, control the total imaging time $<20 \mathrm{~min}$.

\subsubsection{Image processing and analysis}

After the patient's examination is completed, select areas with unclear functions or questionable stuttering and areas that are obviously related to stuttering. Combine the relevant standards in the software library developed by the University of Oxford Brain Function Research Center to determine the corpus callosum, putamen, brain feet, The insula, internal capsule, cerebellum, etc. were identified as the subcortical areas related to stuttering, and the corresponding positions were selected as the ROI. Transfer the obtained data and images to professional software, and define the ratio of FA between the lesion side and the non-lesion side as the FA ratio (rFA). Compare the results of the examination with the results of the clinical examination and diagnose the efficacy. Draw ROC curve and analyze the application efficiency (sensitivity, specificity) of MRI diffusion tensor imaging in patients with stuttering.

\subsection{Statistical analysis}

The SPSS24.0 software was used, counting data with $x^{2}$ test, which is performed by using $\mathrm{n}(\%)$, and the measurement data was performed using $\mathrm{t}$ test, which was represented by $(\bar{x} \pm s)$. The difference at $\mathrm{P}<0.05$ has statistical meaning.

\section{Result}

\subsection{Comparison of rFA between the lesion side and the healthy side of stuttering patients}

70 patients were diagnosed as stuttering patients from clinical examination among the 81 suspected stuttering patients. So the diagnosis rate is $86.42 \%$. All of the patients finished the test of magnetic resonance diffusion tensor imaging, 68 patients were diagnosed as stuttering patients finally. So the diagnosis coincidence rate is $97.14 \%(\mathrm{P}>0.05)$. The $\mathrm{rFA}$ levels of the insula, corpus callosum, and putamen on the side of stuttering lesion are lower than those on the healthy side ( $\mathrm{P}>0.05)$. The rFA of the cerebrum, internal capsule, and cerebellum on the lesion side and the contralateral side has no statistical significance $(\mathrm{P}>0.05)$. Please see Table 1.

Table 1. Comparison of rFA between the lesion side and the healthy side $(\bar{x} \pm s)$

\begin{tabular}{cccccccc}
\hline Type of lesion & Cases & Corpus callosum & Putamen & Cerebral pedunculus & Insula & Inner capsule & Cerebellum \\
\hline Lesion side & 81 & $0.97 \pm 0.13$ & $0.82 \pm 0.16$ & $0.78 \pm 0.13$ & $0.73 \pm 0.16$ & $0.95 \pm 0.18$ & $1.06 \pm 0.21$ \\
Healthy side & 81 & $1.07 \pm 0.16$ & $0.93 \pm 0.19$ & $0.79 \pm 0.14$ & $0.98 \pm 0.17$ & $0.97 \pm 0.20$ & $1.08 \pm 0.23$ \\
t & $/$ & 8.514 & 6.498 & 1.591 & 8.115 & 0.854 \\
P & / & 0.000 & 0.000 & 0.691 & 0.000 & 0.434 & 0.981 \\
\hline
\end{tabular}

\subsection{Application efficacy of magnetic resonance diffusion tensor imaging in stuttering patients}

The ROC curve shows that the AUC data of magnetic resonance diffusion tensor imaging applied in stuttering patients is 0.834 , the diagnostic sensitivity is $84.31 \%$, and the specificity is $71.48 \%$. Please see Figure 1 . 


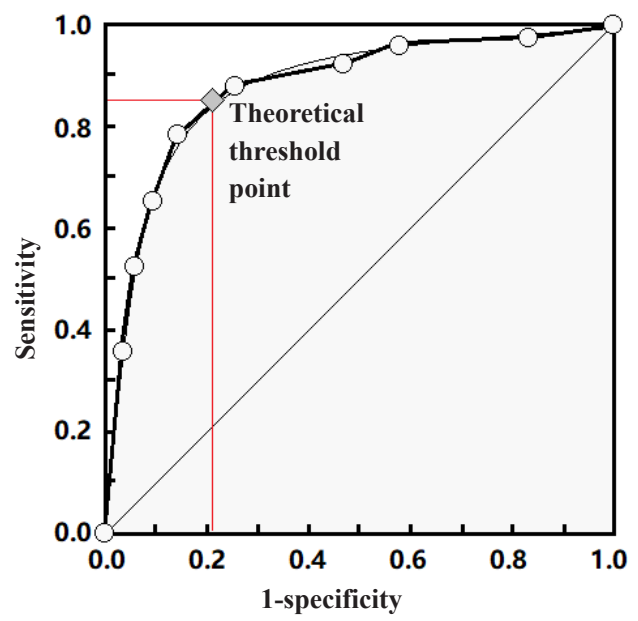

Figure 1. Application of magnetic resonance diffusion tensor imaging in stuttering patients ROC curve

\section{Discussion}

The clinical incidence rage of stuttering is high, and its incidence is mostly related to genetic factors, dysplasia of the language nerve center, neurophysiological genetic and psychological factors, and the pathogenesis of stuttering has not been clarified, which makes clinical diagnosis and treatment very difficult. In recent years, magnetic resonance diffusion tensor imaging has been used in stuttering patients, and the effect is good.

In this research, 70 patients were diagnosed as stuttering patients from clinical examination among the 81 suspected stuttering patients. The diagnosis rate is $86.42 \%$. All of the patients did the test of magnetic resonance diffusion tensor imaging, 68 patients were diagnosed as stuttering patients finally. So the diagnosis coincidence rate is $97.14 \%(\mathrm{P}>0.05)$. The rFA levels of the insula, corpus callosum, and putamen on the side of stuttering lesion are lower than those on the healthy side $(\mathrm{P}>0.05)$. The rFA of the cerebrum, internal capsule, and cerebellum on the lesion side and the contralateral side has no statistical significance $(\mathrm{P}>0.05)$, which indicates that magnetic resonance diffusion tensor imaging can obtain a high diagnostic coincidence rate in stuttering patients, and can provide imaging evidence for clinical diagnosis and treatment. Magnetic resonance diffusion tensor imaging is a special form of magnetic resonance imaging, which is an imaging and postprocessing technology developed and deepened on the basis of magnetic resonance diffusion weighted imaging. Previous studies have shown that: Magnetic resonance diffusion tensor imaging used in stuttering patients can open an important window on structural connectivity, create a three-dimensional space that has anisotropy for water molecule diffusion, and can find the lesion position of stuttering patients and guide clinical treatment ${ }^{[6]}$. In order to further analyzing the application of magnetic resonance diffusion tensor imaging in stuttering patients stuttering, the ROC curve was made in this study. The result shows that the AUC value of magnetic resonance diffusion tensor imaging for patients with stuttering is 0.834 , the diagnostic sensitivity is $84.31 \%$, and the specificity is $71.48 \%$, which indicates that the imaging can improve the diagnostic efficacy of stuttering patients.

In summary, magnetic resonance diffusion tensor imaging can obtain a higher detection rate in stuttering patients and provide imaging evidence for clinical diagnosis and treatment. It is worthy of promotion and application.

\section{References}

[1] Xiao Xiong, Kong Lu, Pan Changcun, et. Application value of diffusion tensor imaging in the surgical treatment of brainstem glioma. Chinese Journal of Neurosurgery. 2019; 35(8): 817-822.

[2] Liu Na, Liu Ni, Geng Deqin, et. Diffusion tensor imaging study on changes of brain structure network in patients with Parkinson's disease and mild cognitive impairment. Chinese Journal of Behavioral Medicine and Brain Science. 2019; 28(3): 193-199.

[3] Jin Wei, Li Yuehua, Libin, et. Research progress of magnetic resonance diffusion tensor imaging and fiber bundle density imaging in the central nervous system. Chinese Journal of Medical Devices. 2019; 43(5): 352-354.

[4] Xie Yunjuan, Liao Lingyi, Gao Qiang. Research progress of diffusion tensor imaging in prognostic evaluation of sports function in stroke patients. Chinese Recovery Theory and Practice. 2019; 25(5): 56-59. 
[5] Liao Lingyi, Xie Yunjuan, Gao Qiang. The research development of diffusion tensor imaging research on the integrity of corticospinal tract and the recovery of motor function after stroke. China Recovery. 2019; 34(9): 48-51.

[6] Chen Siwei, Zhou Lewei, Zhong Hongxi, et. Research on the changes of the white matter structure network of patients with Alzheimer's disease based on diffusion tensor imaging. Chinese Journal of Geriatric Cardiovascular and Cerebrovascular Diseases. 2019; 21(9): 10-14. 